\title{
Planet Populations as a Function of Stellar Properties
}

\author{
Gijs D. Mulders
}

\begin{abstract}
Exoplanets around different types of stars provide a window into the diverse environments in which planets form. This chapter describes the observed relations between exoplanet populations and stellar properties and how they connect to planet formation in protoplanetary disks. Giant planets occur more frequently around more metal-rich and more massive stars. These findings support the core accretion theory of planet formation, in which the cores of giant planets form more rapidly in more metal-rich and more massive protoplanetary disks. Smaller planets, those with sizes roughly between Earth and Neptune, exhibit different scaling relations with stellar properties. These planets are found around stars with a wide range of metallicities and occur more frequently around lower mass stars. This indicates that planet formation takes place in a wide range of environments, yet it is not clear why planets form more efficiently around low mass stars. Going forward, exoplanet surveys targeting $\mathrm{M}$ dwarfs will characterize the exoplanet population around the lowest mass stars. In combination with ongoing stellar characterization, this will help us understand the formation of planets in a large range of environments.
\end{abstract}

\section{Introduction}

Exoplanets are observed around a diverse set of host stars. The first exoplanet discovered around a main-sequence star, 51 Pegasi b, orbits a star enriched in heavy elements (metals) compared to the sun (Mayor and Queloz 1995). In contrast, one of the earliest discovered planets that could conceivably be rocky, Gliese 581e, orbits a metal-poor M dwarf less than a third the mass of the sun (Mayor et al. 2009). While these discoveries represent just two examples of the more than thousand exoplan-

Gijs D. Mulders

Lunar and Planetary Laboratory, The University of Arizona, 1629 E University Blvd, Tucson, AZ 85721, USA, e-mail: mulders lpl.arizona.edu

Earths in Other Solar Systems Team, NASA Nexus for Exoplanet System Science 
ets known to date, they are indicative of the broader trends between exoplanets and their host stars that have since emerged from exoplanet surveys, illustrated in Figure 1. Giant planets occur more frequently around more massive and more metal-rich stars (e.g. Santos et al. 2004; Johnson et al. 2010). Sub-Neptunes occur around stars with a wide range of metallicities (Sousa et al. 2008; Buchhave et al. 2012), but occur more frequently around lower mass stars (Howard et al. 2012; Mulders et al. 2015b).

It is no coincidence that the smallest planets were first discovered around $\mathrm{M}$ dwarfs. The lower stellar mass compared to more sun-like stars with spectral types $\mathrm{F}, \mathrm{G}$ and $\mathrm{K}$ facilitates the detection of less massive planets with radial velocity techniques (e.g. Endl et al. 2003). Similarly, the small size of M dwarfs lead to deeper transits for a planet of the same size when compared to sun-like stars (Dressing and Charbonneau 2013). Most exoplanets to date, however, have been discovered around F, G, and K dwarfs because more bright targets are observable. Hence, an understanding of survey detection efficiency and selection biases are crucial to understand trends in the occurrence of the exoplanet population with host star properties.

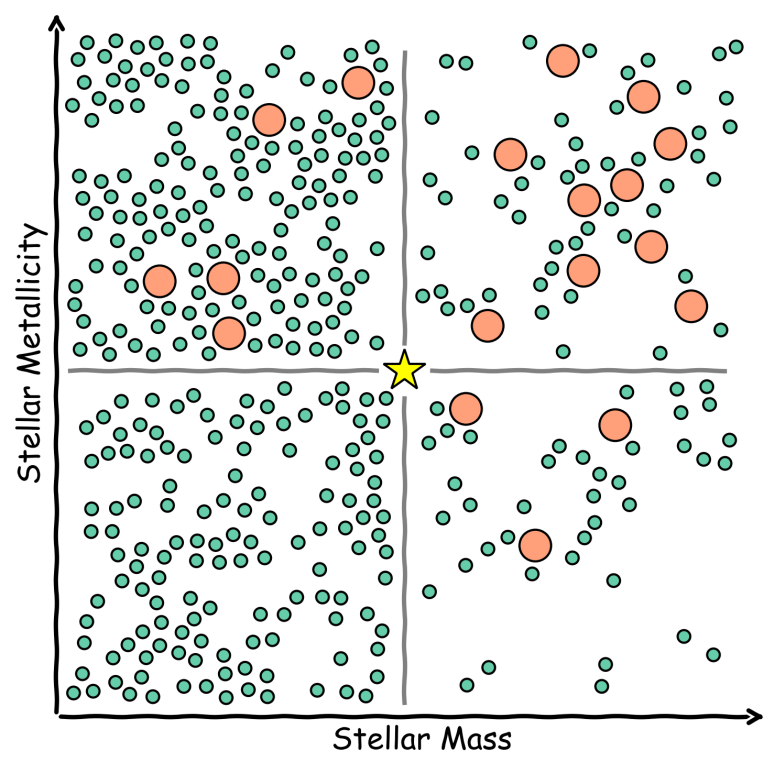

Fig. 1 Trends in the exoplanet population as function of stellar mass and metallicity, illustrating the different behavior of the giant planet population (large pink circles) and planets smaller than Neptune (small cyan circles). The location of the sun is indicated with a yellow star. The location of individual symbols is randomly generated, with the density of point corresponding to the exoplanet occurrence rate. Any resemblance between symbol locations and observed exoplanets is entirely coincidental. 
While the giant planet-metallicity correlation was initially interpreted as pollution of the stellar atmosphere by planetary systems (e.g. Gonzalez|1997), it is now widely accepted that the stellar metallicity is a proxy of the solid inventory of the protoplanetary disks in which planets form. I has been established that the increased occurrence of giant planets around high-metallicity stars arises because giant planet cores are more likely to form in disks with a larger amount of solids (e.g. Ida and Lin 2004). Similarly, the lower frequency of giant planets around $M$ dwarfs can be explained by those stars having less massive disks (Laughlin et al. 2004). The relation between exoplanets and their hosts stars provide stringent constraints on planet formation theory, as properties of exoplanet host stars trace the conditions in protoplanetary disks at the time of planet formation.

These trends, however, breaks down for planets smaller than Neptune, hereafter sub-Neptunes, which poses some urgent questions about the planet formation process. Why is the frequency of sub-Neptunes almost independent of stellar metallicity, even when the initial inventory of condensible solids must have varied by an order of magnitude? Do the elevated planet occurrence rates around M dwarfs, where protoplanetary disk masses were lower, imply that there is something fundamentally different about the planet formation process around low-mass stars?

Not all stars are equally amenable for exoplanets discovery and certain types of stars have been more thoroughly searched than others. To account for these selection and detection biases, planet occurrence rates can be calculated to infer trends in the intrinsic planet population. Variations in the planet occurrence rate with stellar parameters can be estimated from exoplanets surveys under the following conditions:

1. The survey covers a range of stellar properties, with a sufficient number of planet detections across this range to identify trends.

2. Stellar properties are known for the surveyed stars, including those of stars without detected planets, to estimate the fraction of stars with a given set of properties hosting planets.

3. The survey completeness can be estimated, to separate observation bias from intrinsic trends in the exoplanet population.

The focus of this chapter are trends identified in radial velocity and transit surveys with stellar mass and metallicity, which (mostly) satisfy these three requirements.

Trends for giant planets out to a few au and sub-Neptunes at orbital periods shorter than a few hundred days are discussed seperately. Emphasis is placed on studies that take into account the different observation bias and survey detection efficiency that exist when surveying planets around various type of stars. These trends are then placed into the context of planet formation theory and models. An outlook for current and future surveys that can fill in some of the gaps in the current knowledge of the exoplanets populations around different types of stars is presented towards the end of this chapter, followed by a brief conclusion. 


\section{Trends with Stellar Metallicity}

There is a general consensus that giant planet occurrence rates increase with host star metallicity, see also the review by Udry and Santos (2007). The giant planetmetallicity relation is seen in radial velocity surveys of sun-like stars, $M$ dwarfs, and evolved stars, and has also been identified for transiting planets in the Kepler survey. However, Sub-Neptunes are found around stars with a wider range of metallicities, with no clear preference for metal-rich stars. Throughout this chapter, the logarithm of the iron abundance with respect the solar abundance, $[\mathrm{Fe} / \mathrm{H}]$, is used to represent stellar metallicity.

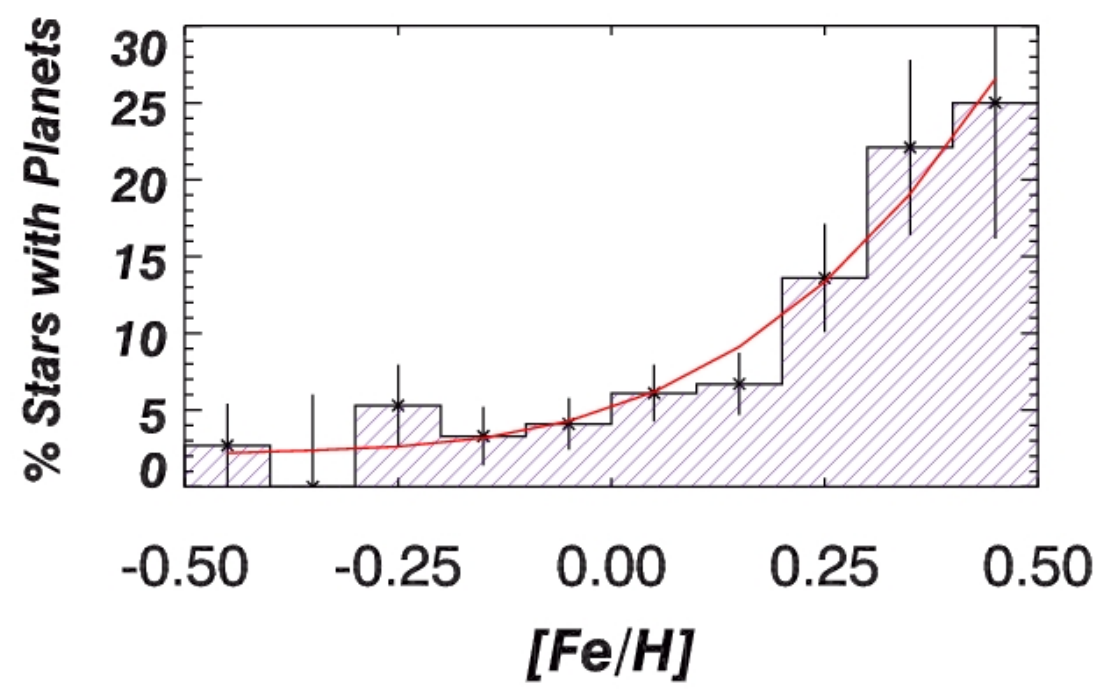

Fig. 2 Giant planet occurrence rate as function of stellar metallicity, from Fischer and Valenti (2005) figure 5. The red solid line shows a quadratic relation between planet occurrence and stellar metallicity $(\beta=2$, eq. 1 1 . Figure reproduced from Fischer and Valenti (2005) with permission from the authors.

\section{Positive Giant Planet-Metallicity Correlation}

Giant planets occur more frequently around stars with higher metallicities (See Fig. 2). The first indications of a planet-metallicity correlation were found by Gonzalez (1997) and Marcy et al. (1997) based on metallicities of a handful of exoplanet hosts including 51 Peg b. The trend that giant planets are preferentially found around 
metal-rich host stars was subsequently recovered in larger samples (Gonzalez 1998, Fuhrmann et al.|1998; Santos et al.2000; Gonzalez et al.2001; Sadakane et al.2002, Laws et al. 2003).

As outlined in the preceding section, characterizing star properties of non-planet hosts and detection efficiency of the survey are critical to separate observation bias from intrinsic planet population. Santos et al. (2001, 2003) measured abundances for non-planet hosting stars and found that the giant planet hosts are systematically more metal-rich in a volume-limited sample. The detection frequency of giant planets was shown to increase with metallicity in a volume-limited sample of stars from the Hipparcos catalog (Reid 2002). Santos et al. (2004) estimated planet occurrence rate as function of metallicity and identified a positive correlation at super-solar metallicities. The high occurrence rate of giant planets around metal-rich stars was confirmed by (Fischer and Valenti 2005, see also Fig. 2), who derived stellar abundances of stars in the Keck, Lick, and Anglo-Australian Telescope planet search surveys.

The occurrence rate of giant planets is a strong function of metallicity and scales roughly with the square of the number of iron atoms. At super-solar metallicities, $[\mathrm{Fe} / \mathrm{H}]>0$, where planet detections are plenty, metallicity increases by a factor of 5 from $\sim 5 \%$ at $[\mathrm{Fe} / \mathrm{H}]=0$ to $\sim 25 \%$ at $[\mathrm{Fe} / \mathrm{H}]=0.5$. At lower metallicities, the shape of the metallicity distribution is less well quantified due to few planet detections, with a giant planet occurrence rate of approximately $\sim 2-3 \%$ (e.g. Santos et al. 2004). The functional form of the planet occurrence-metallicity correlation is often assumed to be a power-law 1

$$
f_{\text {giant }} \propto 10^{\beta[\mathrm{Fe} / \mathrm{H}]},
$$

with index $\beta \approx 2$ (e.g. Fischer and Valenti 2005; Udry and Santos 2007; Sousa et al. 2011). Johnson et al. (2010) showed that such a functional form provides a better fit than a flat distribution at sub-solar metallicities. The planet occurrence rate likely continues to decrease at metallicities below $[\mathrm{Fe} / \mathrm{H}]<-0.5$ (Santos et al. 2011; Mortier et al. 2012), consistent with the non-detection of giant planets in metal-poor clusters (e.g. Gilliland et al.|2000). However, Mortier et al. (2013a) also argue that planet statistics at low metallicity are too small to discriminate between a linear function and a power-law.

Transiting Giant Planets The planet-metallicity correlation has also been identified for transiting planets in the Kepler survey. The biggest challenge in identifying this correlation lies in characterizing stellar properties, in particular for non-planet host stars. The volume of stars searched for transiting planets is much larger than in radial velocity surveys - both in terms of absolute numbers and galactic distance and characterization of stellar properties with high spectral resolution observations requires a significant investment in observing time. For this reason, most surveys have focused on characterizing planet-hosting stars (Everett et al. 2013, Buchhave et al. 2014; Johnson et al. 2017). At time of writing, the stellar properties of the

\footnotetext{
${ }^{1}$ Note that $[\mathrm{Fe} / \mathrm{H}]$ is the logarithm of the iron abundance.
} 
majority of Kepler targets are based on broad-band photometry (e.g. Mathur et al. 2017), where the derived metallicity is unreliable (Brown et al.|2011, see also Gaidos and Mann 2014: Huber et al. 2014) and giant planet occurrence rates as a function of spectroscopic metallicity have not yet been calculated.

A giant planet-metallicity relation in the Kepler survey was first identified based on photometry by Schlaufman and Laughlin (2011), who find that giant planet hosts have systematically redder colors than non-planet hosts, consistent with a metallicity increase in 0.2 dex. Spectroscopic characterization of exoplanet host stars show that planets larger than $4 R_{\oplus}$ are preferentially found around stars with a supersolar metallicity of 0.15-0.18 dex (Buchhave et al. 2012, 2014; Winn et al. 2017). This result was confirmed by Everett et al. (2013) who found that giant planets only occur around high-metallicity stars $([\mathrm{Fe} / \mathrm{H}]>-0.05 \mathrm{dex})$. Wang and Fischer (2015) foundnd 10 times more planets around metal-rich stars based on photometric metallicities, consistent with a power-law index $\beta=2$ as found in radial-velocity surveys. Medium-resolution spectroscopic metallicities from LAMOST also show a similar metallicity increase of $\sim 0.14$ dex for giant planet hosts (Mulders et al. 2016).

Dwarfs and Giants The giant planet-metallicity correlation is also present in stars with lower and higher masses than the sun. Low-mass $\mathrm{M}$ dwarfs $\left(\lesssim 0.5 M_{\odot}\right)$ are found to be enhanced in metallicity when they host giant planets (Bonfils et al. 2007; Johnson and Apps 2009; Rojas-Ayala et al. 2012, Terrien et al. 2012). The exponent of the occurrence rate-metallicity correlation, in the range $\beta=[1.26,2.94]$, is consistent with that of sun-like stars (Neves et al. 2013). The planet-metallicity correlation is less statistically robust than for FGK dwarfs due to a lower number of planet detections (Schlaufman and Laughlin|2010 Gaidos and Mann|2014).

Giant and sub-giant stars that have evolved off the main sequence provide an opportunity to measure planet occurrence rates around higher mass stars $\left(\gtrsim 1.5 M_{\odot}\right)$. The giant planet-metallicity correlation is less well established for these evolved stars than for main-sequence stars. Hekker and Melendez (2007) found the first indications that evolved planet hosts are more metal-rich than non-planet hosts. Subsequent studies did often not find a planet-metallicity correlation (Pasquini et al. 2007; Takeda et al. 2008; Mortier et al.|2013b), showed mixed results (Maldonado et al. 2013, Jofré et al. 2015), or did recover a correlation (Wittenmyer et al.|2017). Limiting this chapter to planet occurrence rate studies, i.e. those that take into account detection efficiency and sample selection, the planet occurrence rate is found to increase with stellar metallicity (Johnson et al. 2010; Reffert et al. 2015; Jones et al. 2016).

\section{A Wide Range of Stellar Metallicity for sub-Neptunes}

Planets smaller than Neptune form around stars with a wide range of metallicities (Sousa et al. 2008, Buchhave et al. 2012). The planet-metallicity correlation identi- 


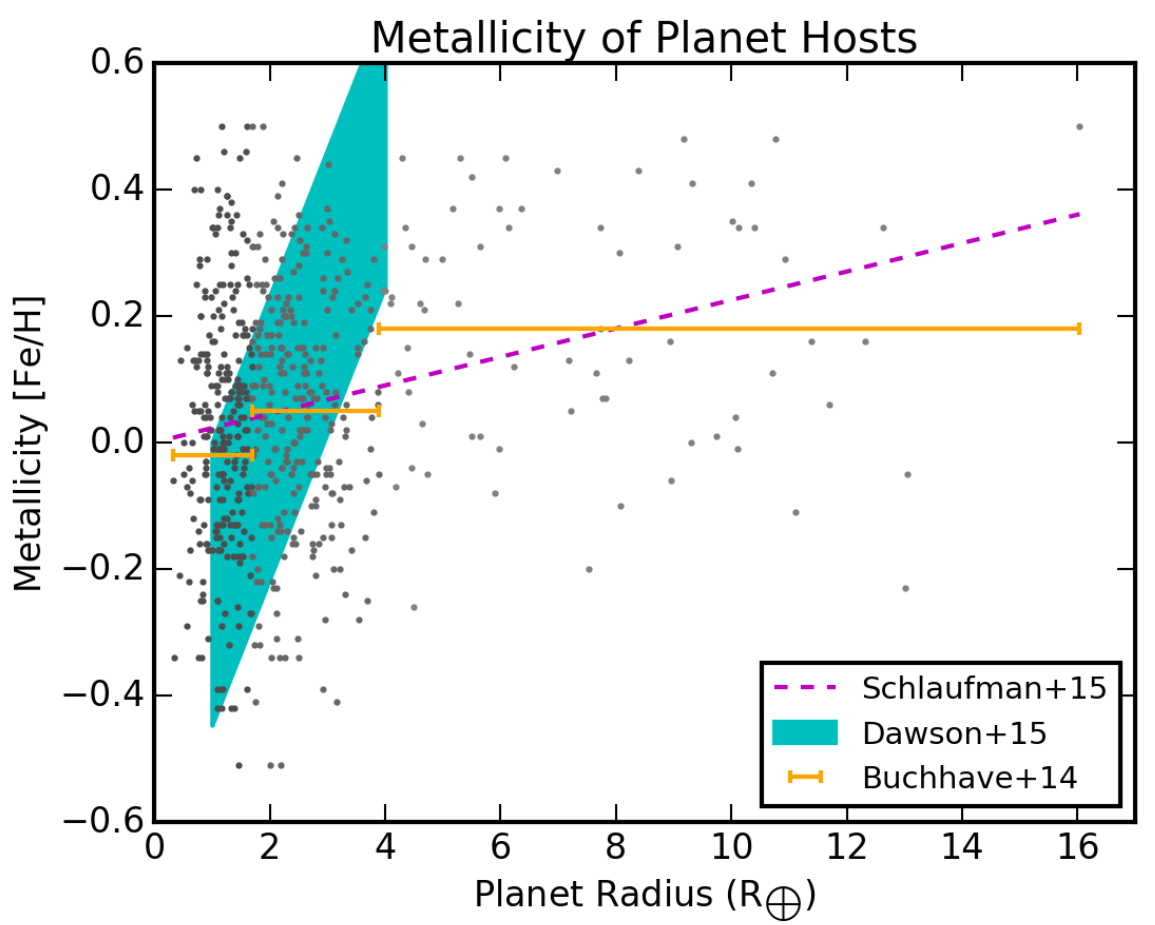

Fig. 3 Metallicity of planet host stars as function of planet radius. Points represent spectroscopic metallicities of Kepler exoplanet hosts from Buchhave et al. (2014). The average host star metallicity correlates with planet radius, as indicated for a set of discrete radius bins shown in orange (Buchhave et al. 2014) and for a continuous planet radius-metallicity relation (Schlaufman 2015) shown with the dashed purple line. The expected range of planet radii from In Situ planet formation models by Dawson et al. (2015) are shown in cyan.

fied for giant planets disappears when considering smaller planets (Fig 3, Buchhave et al.2014).

Neptunes The first indications that Neptune-mass planets are not preferentially found around metal rich stars, as opposed to giant planet hosts, were found by Udry et al. (2006) in a sample including $M$ dwarfs planet hosts, and later confirmed by Sousa et al. (2008). The possibility that a higher planet occurrence rate of Neptune-sized planets around $\mathrm{M}$ dwarfs contributed to this correlation was investigated by Ghezzi et al. (2010), who recovered the wide range of stellar metallicities for Neptune-mass planet hosts in a sample of FGK dwarfs. This trend was confirmed by Mayor et al. (2011), who show that planets less massive than $30-40 M_{\oplus}$ are equally common around metal-poor and metal-rich stars. The same metallicityindependence was found for $\mathrm{M}$ dwarfs hosting Neptune mass and smaller planets (Rojas-Ayala et al.|2012, Neves et al.|2013). 
Transiting sub-Neptunes The large number of planets smaller than Neptune discovered by the Kepler mission provide a unique opportunity to constrain the metallicity-dependence of planets down to Earth-sizes. Follow-up high resolution spectroscopy of Kepler exoplanet hosts confirm that sub-Neptunes form around a wide range of stellar metallicities $([\mathrm{Fe} / \mathrm{H}] \approx[-0.6-0.5])$ and extend this trend to Earth-sized planets (Buchhave et al. 2012; Everett et al. 2013; , Mann et al.|2013).

Buchhave et al. (2014) divided the sample into rocky planets $\left(R<1.7 R_{\oplus}\right)$ and gas dwarfs $\left(1.7 R_{\oplus}<R<3.9 R_{\oplus}\right)$ and find that the mean metallicity of rocky planets is consistent with solar. On the other hand, the larger gas dwarfs have a mean metallicity of $[\mathrm{Fe} / \mathrm{H}]=0.05$ that is significantly higher than non-planet hosting stars (Buchhave and Latham 2015). Such a trend is consistent with a planet-metallicity correlation for the maximum size/mass of Neptunes (Courcol et al. 2016, Petigura et al. 2017b). However, Schlaufman (2015) argue that the Kepler data is better described by a continuous increase in metallicity with planet radius (Figure 3 .

Planet occurrence rates as a function of spectroscopic metallicity were calculated by Mulders et al. (2016) for a sample of 20,000 Kepler target stars with medium resolution spectroscopy from Frasca et al. (2016). They find no difference in the occurrence rate of sub-Neptunes as a function of metallicity, except at orbital periods smaller than 10 days (see also Wilson et al. 2017; Petigura et al.|2017a). This elevated occurrence rate at short orbital periods is consistent with the higher detection frequency of sub-Neptunes around metal-rich stars (Wang and Fischer 2015, Zhu et al. 2016).

Several other papers have pointed out trends in host star metallicity with the planet orbital period distribution (Beaugé and Nesvorný 2013, Adibekyan et al. 2013: Dawson et al. 2015: Dong et al. 2017), though there is some disagreement on the planet radius and orbital period where these transitions occur. The trend identified by Adibekyan et al. (2016) that small $\left(<2 R_{\oplus}\right)$ planets interior to the habitable zone may predominantly found in low-metallicity stars is tantalizing, but was found to not be significant when taking into account detection completeness by Mulders et al. (2016).

\section{Trends With Stellar Mass}

The correlation between planet occurrence and stellar mass is dependent on planet size (Fig. 55). Giant planets occur more frequently around higher-mass stars (Fig. 4. Johnson et al. 2010), but the dependence is weaker than that with metallicity and less statistically significant. Sub-Neptunes, those found in abundance with the Kepler survey, occur more frequently around low-mass M dwarfs (Fig 6, Mulders et al. 2015c). 


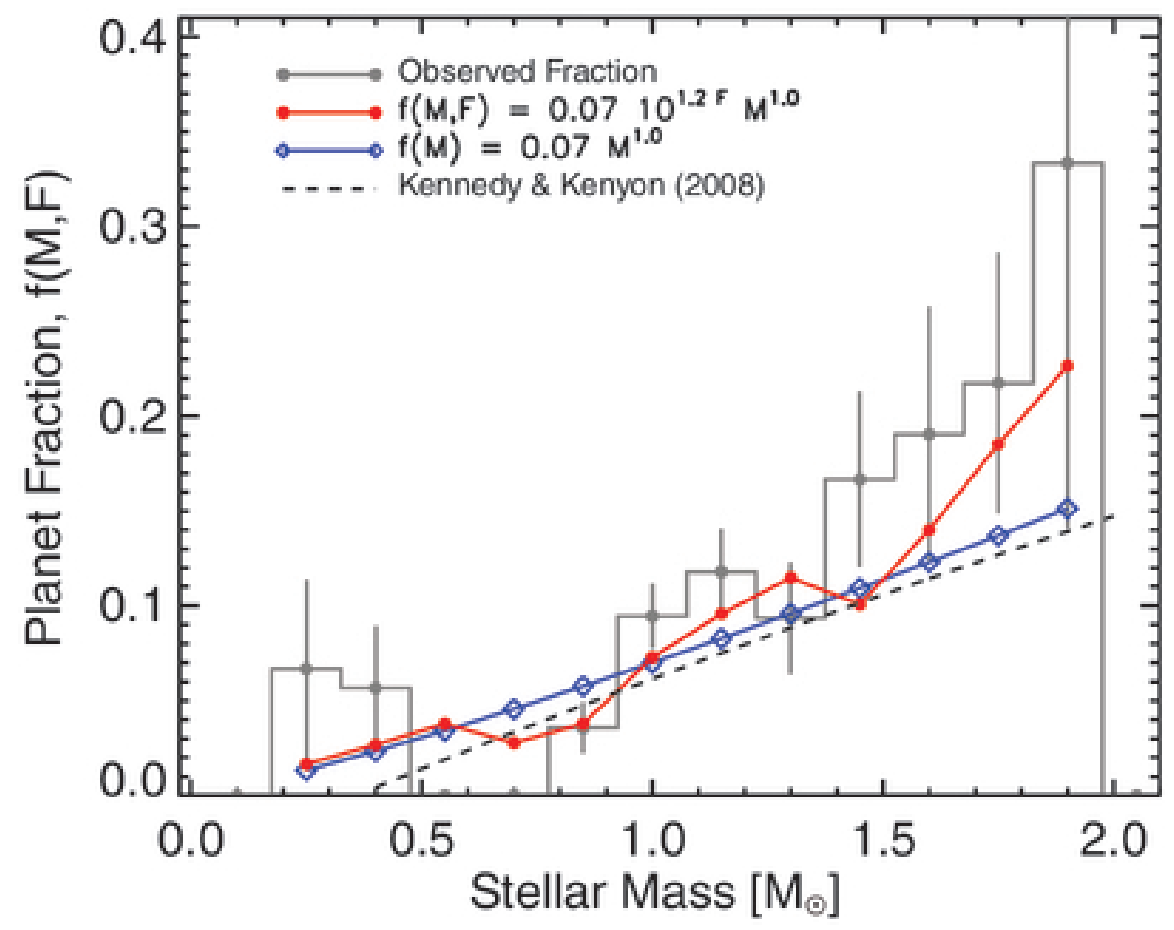

Fig. 4 Giant planet occurrence as function of stellar mass, from Johnson et al. (2010) figure 4. The histogram shows the observed planet occurrence rate. The red red line show the predicted planet occurrence rate based on the metallicity distribution of stars in each stellar mass bin. The blue line shows the stellar-mass dependence at solar metallicity, compare to the predicted relation from the planet formation model by Kennedy and Kenyon (2008). Figure reproduced from Johnson et al. 2010) with permission from the authors.

\section{Giant planets}

Giant planets are found less frequently are low-mass $\mathrm{M}$ dwarfs than around sun-like stars and more frequently around evolved stars with higher masses (Fig. 4). However, the presence of a giant planet-stellar mass correlation is less well established than the planet-metallicity correlation. The main challenge lies in correcting for the planet-metallicity correlation, which is stronger $\left(f_{\text {giant }} \propto[\mathrm{Fe} / \mathrm{H}]^{2}\right)$ than the planetmass correlation $\left(f_{\text {giant }} \propto M_{\star}\right)$.

Tentative evidence for a decreased giant planet occurrence around $\mathrm{M}$ dwarfs compared to sun-like stars was found by Laws et al. (2003) and Endl et al. (2006). The giant planet occurrence rate within 2.5 au increases by a factor of $\sim 3$ from $\mathrm{M}$ stars to sun-like stars (Butler et al. 2006, Cumming et al. 2008). Planet occurrence rates for a sample of late $\mathrm{K}$ dwarfs support the positive correlation with stellar mass (Gaidos et al.2013). 
Taking metallicity into account, the giant planet occurrence rate increases roughly linear with stellar mass between M dwarfs, GK stars, and retired A stars (Johnson et al. 2007, 2010, Fig. 4). The stellar-mass dependence has also been identified for giant planets at longer orbital periods by including radial-velocity trends and microlensing data (Montet et al. 2014, Clanton and Gaudi 2014). However, Mortier et al. (2013a) and Gaidos and Mann (2014) show that the lower occurrence rate around M dwarfs compared to sun-like stars is not statistically significant: the data are consistent with both a linear or no dependence on stellar mass. No residual trends with stellar mass are found within radial velocity samples of sun-like stars (Fischer and Valenti 2005) or M dwarfs (Neves et al. 2013). The planet occurrence rate around giant stars increases with stellar mass up to $2 M_{\odot}$ but decreases at larger stellar mass (Reffert et al. 2015; Jones et al. 2016).

The giant planet occurrence rate in the Kepler transit surveys is low, consistent with the predictions from radial velocity surveys (Dressing and Charbonneau 2013). The occurrence rate of giant planets with orbital periods less than 50 days is more than two times higher for FGK stars than M stars (Mulders et al. 2015c). Groundbased transit surveys do not yet have the statistics to confirm the lower occurrence rate of giant planets around $\mathrm{M}$ stars (Obermeier et al. 2016), though new discoveries provide opportunities to constrain their occurrence rate (Bayliss et al.|2017).

\section{The Sub-Neptune Exoplanet Population}

Planet occurrence rate increases steeply towards lower-mass planets around sunlike stars (e.g. Howard et al.|2010, 2012). The shape of the planet radius distribution is dependent on stellar mass: the planet occurrence rate increases even steeper for low-mass M dwarfs (Figure 5, Mulders et al. 2015c). Neptune-mass and smaller planets are commonly found around $\mathrm{M}$ dwarfs in radial velocity surveys, where the smaller mass ratio between star and planet favors planet detection compared to FGK stars. The sub-Neptune exoplanet population is most constrained by Kepler, whose detection efficiency reaches down to earth radii and smaller at short orbital periods.

High planet occurrence around low-mass stars The increase in exoplanet occurrence with decreasing effective temperature (a proxy of stellar mass) was discovered by Howard et al. (2012). Taking into account differences in detectability between stars of different sizes in the Kepler survey, they find that the occurrence rate of planets between 2-4 $R_{\oplus}$ is anticorrelated with effective temperature and increases by a factor 7 between the hottest stars in the sample (late F stars) and the coolest stars (late $\mathrm{M}$ dwarfs). This trend was extended down to Earth-sized planets by Mulders et al. (2015b), who found an increase in planet occurrence rate between F,G,K, and $\mathrm{M}$ type stars at all orbital periods. The occurrence rate of (late) $\mathrm{M}$ stars compared to FGK stars is a factor $\sim 2-4$ higher at planet radii between 1 and $\sim 3 R_{\oplus}$ (Mulders et al. 2015c; Gaidos et al.2016). At larger planet radii $\left(>4 R_{\oplus}\right)$, the trend reverses and planets become more common around sun-like stars (Figure 5) as dis- 


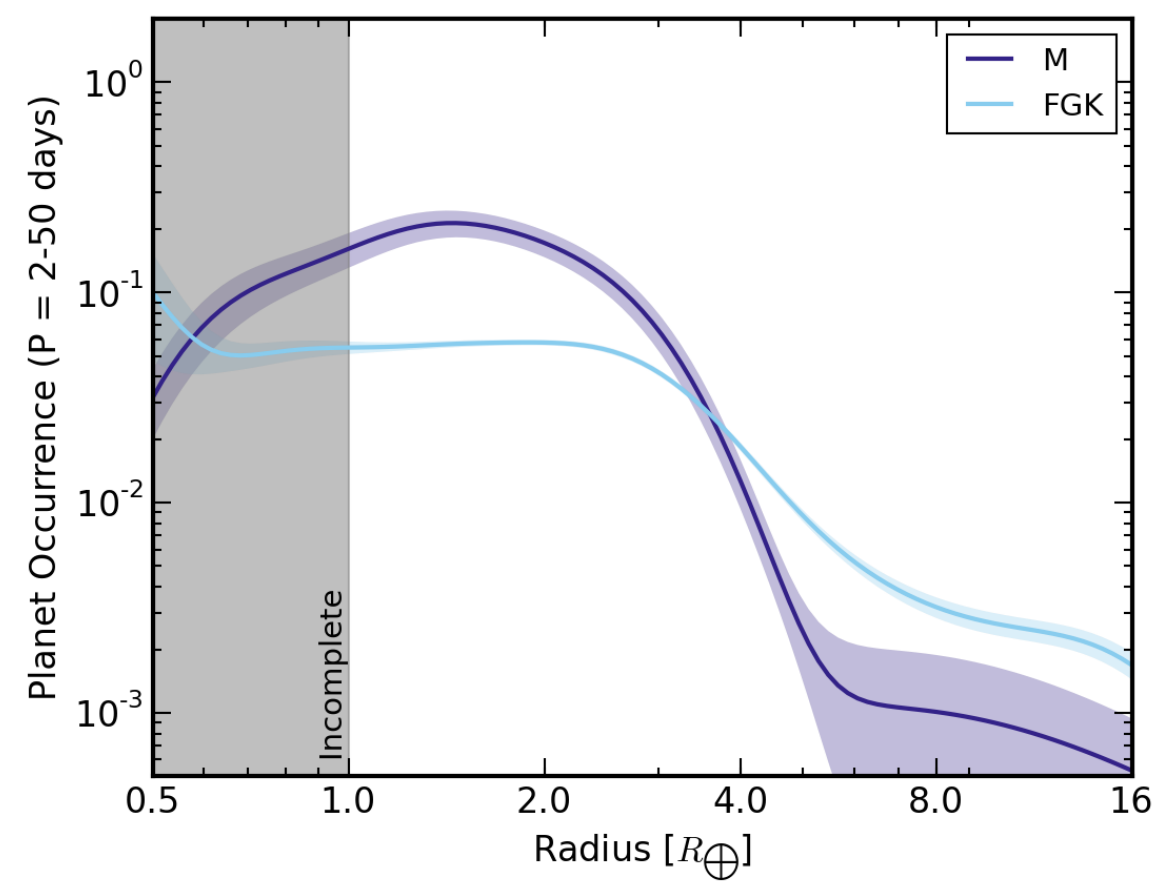

Fig. 5 Debiased planet radius distribution of exoplanets around $\mathrm{M}$ dwarfs (purple) and FGK stars (cyan), from Mulders et al. (2015c) Figure 5. Planets smaller than $2.8 R_{\oplus}$ are 3.5 times more abundant around $\mathrm{M}$ stars, while giant planets occurrence is at least a factor 2 higher around FGK stars.

cussed before. This trend is not a result of selection and detection biases as briefly discussed below.

Detection biases Occurrence rate calculations take into account planet detection efficiency as function stellar properties such as stellar size and noise level. At this point it is worth noting that many occurrence rate studies employing different methodologies have been conducted on the Kepler sample of $M$ dwarfs that generally find good agreement on planet occurrence rates (Dressing and Charbonneau 2013: Morton and Swift 2014, Dressing and Charbonneau 2015, Mulders et al. 2015c Gaidos et al. 2016). Comparison with occurrence rate studies around sun-like stars can be made - though one has to keep in mind that different treatment of detection efficiency can affect occurrence rate estimates (e.g. Christiansen et al.|2015, Burke et al.2015). Figure 6 shows the occurrence of rate of sub-Neptunes $\left(1-4 R_{\oplus}\right)$ at orbital periods less than $\mathbf{5 0}$ days as a function of stellar effective temperature as estimated by different studies. For purposes of this comparison, occurrence rates were rescaled when only estimates for a different range of planet properties were available, assuming a uniform occurrence in log planet radius and log orbital period. While there is significant scatter in occurrence rates at similar effective tem- 
peratures, the elevated planet occurrence rates around $\mathrm{M}$ dwarfs compared to FGK stars is clearly present.

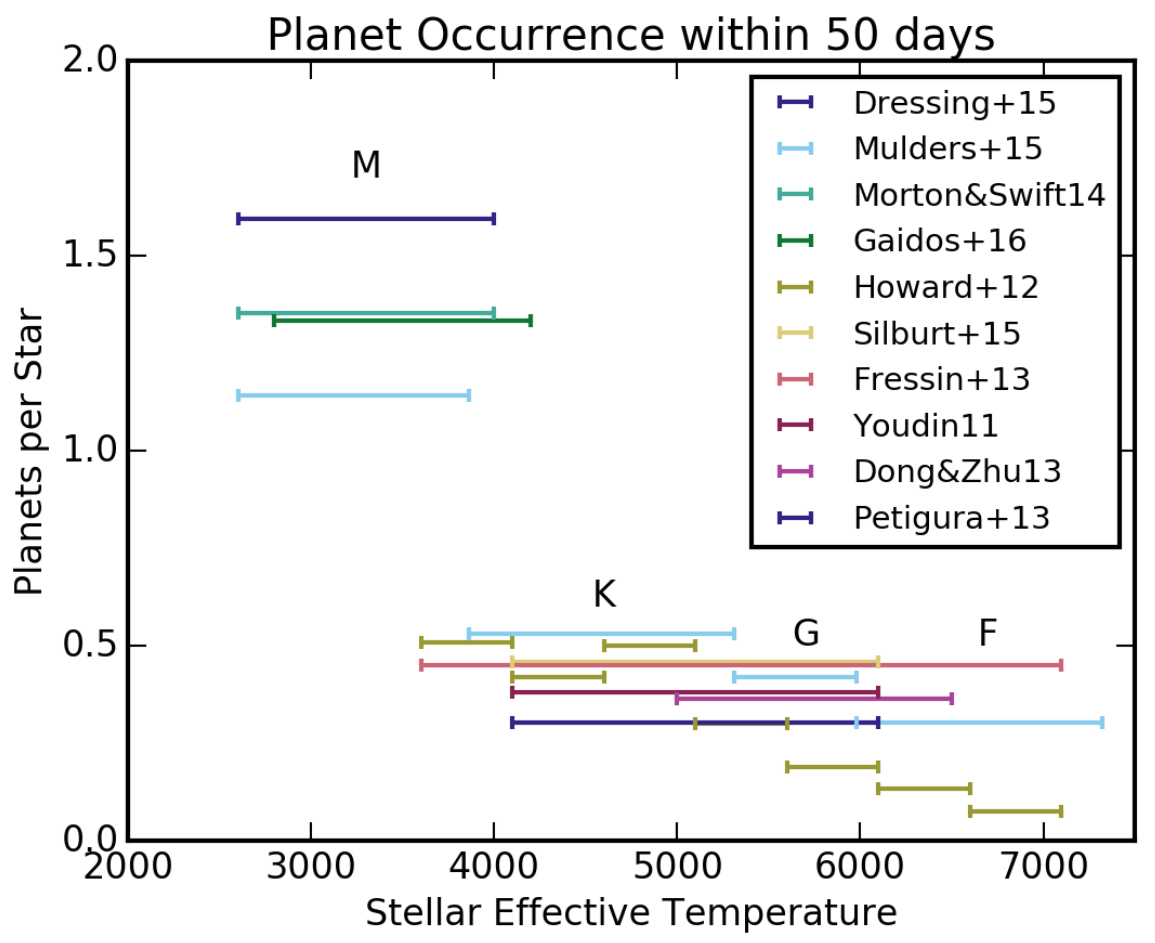

Fig. 6 Overview of planet occurrence rates as a function of effective temperature in the literature for planets between $1-4 R_{\oplus}$ and $P<50$ days. Occurrence rates were re-scaled assuming uniform occurrence in $\log$ period and $\log$ radius for purpose of this comparison. The occurrence rates for low-mass M dwarfs are systematically higher than those of FGK stars. Two studies (Howard et al. 2012. Mulders et al.2015c) also show trends within the sample of F,G, and $\mathrm{K}$ stars. References Dressing and Charbonneau (2015); Mulders et al. (2015c); Morton and Swift(2014); Gaidos et al. (2016); Howard et al. (2012); Silburt et al. (2015); Fressin et al. (2013); Youdin (2011); Dong and Zhu (2013); Petigura et al. (2013).

Selection effects Because Kepler is a magnitude-limited survey, more luminous stars can be detected at larger distances. The observed population of more massive stars is therefore, on average, more distant from the sun and higher above the galactic plane, and may probe a stellar population that may be older and lower in metallicity. Future and ongoing transit surveys may quantify the effect of different galactic locations on planet occurrence rates. The differences in the distribution of stellar metallicities between M dwarfs and FGK stars are small. Howard et al. (2012) show that, based on galactic stellar models, the expected differences in mean metallicity between stars of different spectral types probed with Kepler is less than 
0.1 dex. Spectroscopic metallicities for the Kepler $\mathrm{M}$ dwarf sample are consistent with the metallicity distribution in the solar neighborhood (Mann et al. 2013). The average metallicity of sun-like stars in the Kepler field appears to be sub-solar (e.g. Dong et al.2014), but the metallicity difference is too small to have a large impact on the giant planet population (e.g Guo et al. 2016). For smaller planets, in particular those orbiting $\mathrm{M}$ dwarfs, the occurrence rate does not have a strong dependence on metallicity (Mann et al.|2013, Gaidos et al.|2016). Therefore a different distribution of stellar metallicity is unlikely to explain the elevated planet occurrence rates of M dwarfs.

\section{Constraints On Planet Formation Mechanisms}

The dependence of the exoplanet population on host star properties provides constraints on planet formation mechanisms. The positive correlations of giant planet occurrence rate with stellar mass and metallicity support the core accretion scenario of giant planet formation. The constraints provided by the lack of a clear correlation for sub-Neptunes with stellar metallicity and the anti-correlation with stellar mass have yet to be determined. These trends indicate that planet formation is a robust and efficient process that takes place in a variety of environments.

\section{Formation of Giants Planets}

The core accretion scenario postulates that giant planets form "bottom up" with the formation of $\mathrm{a} \sim 10 M_{\oplus}$ solid core followed by a subsequent phase where most of the gas is accreted (Pollack et al. 1996). As the envelope has to be accreted before the protoplanetary disk gas is dispersed, typically $\sim 3$ million years (e.g Mamajek 2009), the growth of the core has to be sufficiently rapid to allow giant planets to form. The time scale for core growth depends on the amount of material locally available in the disk, i.e. the solid surface density. Giant planets form only in protoplanetary disks with a sufficiently high surface density of solids (e.g. Ikoma et al. 2000; Kokubo and Ida 2002).

The stellar metallicity is a tracer of the solid inventory in protoplanetary disks at the onset of planet formation. Stars and protoplanetary disks inherit the same metallicity from the parental molecular cloud. Stars with a high metallicity formed with disks with a high solid surface density, and are therefore more likely to form giant planets. Numerical simulations of core formation and envelope accretion in disk with different metallicities consistently reproduce the observed giant planetmetallicity correlation (e.g. Ida and Lin 2004; Kornet et al.|2005; Ida and Lin 2008, Mordasini et al.|2009b).

A similar argument can be made for the dependence of the giant exoplanet population on stellar mass. Protoplanetary disks mass, both gas and solids, scales with 
stellar mass (see Fig. 77), while giant planets are more likely to form in more massive disks (e.g. Thommes et al. 2008). By extension, the core accretion model predicts a positive correlation between giant planet occurrence and stellar mass. Based on analytical estimates, Laughlin et al. (2004) predict fewer giant planets around M dwarfs. Detailed numerical simulations show a nearly linear dependence of giant planet occurrence on stellar mass (Ida and Lin 2005, Kennedy and Kenyon 2008, Alibert et al. 2011), consistent with the observed trends (Figure 47.

Gravitational instability In the gravitational instability scenario, giant planets form "top down" from the contracting gas in massive protoplanetary disks (Boss 1997). This formation mechanism predicts different dependence on stellar mass and metallicity. A high disk metallicity inhibits cooling and contraction of the gaseous envelope, and therefore giant planets should form more efficiently around lowmetallicity stars (Meru and Bate 2010). Gravitational instabilities should also form planets efficiently around M dwarfs (Boss 2006). The observed positive correlations between giant planet occurrence with stellar mass and metallicity indicate that planets at short orbital periods likely did not form through gravitational instability in a protoplanetary disks.

Increasing Stellar Metallicity by Accretion of Planets Accretion of planets can increase the stellar metallicity if planets are more metal-rich than their host star. It was initially suggested that the enhanced metallicity of planet-hosting stars is caused by the accretion of planets or solids (Gonzalez 1997), instead of planet formation being more efficient around more metal-rich stars. The observational signature of planetary accretion is only large enough if the accreted metals are not mixed throughout the entire star, but remain near the surface in the convective zone. In F and A stars, the convective zone is thin enough that the accretion of solids can lead to a metallicity increase that is consistent with observations (Laughlin and Adams 1997). For lower-mass stars the convective zones are deeper and the metallicity signature of accreted planets should drop below detectable levels for $\mathrm{G}$ type and earlier stars (Laughlin and Adams 1997). This prediction is inconsistent with the observed giant-planet metallicity relation for these stars (e.g. Fischer and Valenti 2005) as well as for M dwarfs (e.g. Neves et al. 2013). Once stars evolve off the main-sequence, mixing should increase, thereby diluting the metallicity enhancement from planetary accretion. However, the planet metallicity correlation is also observed in evolved stars (e.g. Johnson et al. 2010; Reffert et al. 2015; Jones et al. 2016). Hence, the hypothesis that planetary accretion causes the planet-metallicity correlation is no longer supported by observational evidence.

\section{Formation of sub-Neptunes}

The different scaling laws with stellar mass and metallicity indicate a different formation history for giant planets and sub-Neptunes. Indeed, the comparison between the predictions of the core accretion model (Lin 2008, Mordasini et al.2009a) with 
the population of sub-Neptunes detected in radial velocity surveys (Howard et al. 2010) and the Kepler transit survey (Howard et al. 2012) show that the predicted "planet desert" at orbital period less than 50 days is indeed well-populated, highlighting the need to amend planet formation theory for sub-Neptunes.

The moniker of 'core accretion' is not particularly useful when discussing subNeptunes as they are, almost by definition, the planets that did not accrete massive gaseous envelopes. The planet formation mechanisms discussed here are almost exclusively focused on sub-Neptunes and it should be kept in mind that these new mechanisms are to amend, not replace, core accretion theory.

Several planet formation mechanisms have been proposed to explain the presence of small planets at short orbital periods (e.g. Raymond et al. 2008). The two mechanisms that are of most relevance here are In Situ formation and Planet Migration.

In Situ Formation The In Situ Formation scenario for exoplanets is based on terrestrial planet formation in the Solar System. Planetary embryos in the protoplanetary disk can grow through oligarchic growth to a fraction of the final planet mass, typically Mars-size at 1 au (e.g. Lissauer 1987; Kokubo and Ida 2000, 1998) After the gas disk disperses, gravitational interactions increase the protoplanet eccentricities and makes them collide and merge, leading to a phase of giant impacts in which planets grow to their final masses (e.g. Chambers and Wetherill 1998, Wetherill 1985). As the majority of the accreted material is sourced from a region close to the planets final orbit, the planet mass is directly dependent on the local surface density of planetary building blocks (Kokubo and Ida 2002). Chiang and Laughlin (2013) proposed that planetary systems observed with Kepler could have formed In Situ in disks that are on average more massive than the protoplanetary disk around the sun. N-body simulations of the giant impact phase show that disks with high surface density of solids in the inner regions can indeed form Kepler-like planetary systems (Hansen and Murray|2012, 2013). The main criticism of the In Situ planet formation model is that it is not clear if the inner regions of protoplanetary disks can indeed contain enough mass that grow into planetary embryos (Schlichting 2014).

Planet Migration The Planet Migration hypothesis is built on he theoretical expectation that low-mass planets embedded in a gaseous disk undergo rapid inward migration (Type-I migration, Ward 1997). Because planetary embryos can grow to larger sizes in the outer disk where more material is available, Planet Migration does not require disks to be particularly massive (e.g. Swift et al.|2013). The type-I migration time scales are short $\left(<10^{5}\right.$ years) compared to the disk life time of a few million years (e.g. Mamajek 2009), and migration needs to be halted in the inner disk. Possible mechanisms to stall migration include an inner disk cavity (e.g. Terquem and Papaloizou 2007), resonant capture by other planets, and regions of outward migration due to disk density and temperature structure (Dittkrist et al. 2014; Cossou et al. 2014). The largest challenge for planet migration hypothesis is that the observed multi-planet systems are often not in orbital resonances as predicted from convergent migration (Fabrycky et al. 2014), though different mecha- 
nisms have been proposed to break resonances after formation (Rein 2012, Goldreich and Schlichting 2014; Izidoro et al. 2017).

These two scenarios are not mutually exclusive. Planet Migration models often include a a giant impact phase during or after migration. In Situ formation models often invoke, explicitly or implicitly, an inward migration phase of solids to increase the amount of planetary building blocks in the inner disk (e.g. Hansen and Murray 2012). Despite these nuances, Planet Migration and In Situ remain useful concepts in discussing the origin the observed trends with stellar mass and metallicity.

Metallicity Dependence The stellar metallicity is a direct measure of the amount of condensible solids that was available for planet formation in the disk. The base expectation is that the mass in planetary systems correlates positively with disk metallicity. The In Situ formation simulations in Dawson et al. (2015) show that, for a range of metallicity of a factor 10 , the predicted planet radii vary between $1-4 R_{\oplus}$, with significant scatter (see also Figure 33. A clear planet size-metallicity relation is not seen in the observed population of small exoplanets. There is tentative evidence for a lack of rocky $\left(<2 R_{\oplus}\right)$ planets at high metallicities at a limited orbital period range (Dawson et al. 2015, Adibekyan et al.|2016), though this trend may not be statistically significant when taking into account survey completeness (Mulders et al. 2016). The predicted lack of sub-Neptunes $\left(2-4 R_{\oplus}\right)$ at low metallicity is not observed. However, a planet size-metallicity relation appears to be present for planets more massive than Neptune (Courcol et al. 2016; Petigura et al. 2017b). The elevated host star metallicity of transiting sub-Neptunes (Buchhave et al. 2014, Buchhave and Latham 2015) seems to support In Situ formation scenario, perhaps with a much wider range in planet radii than predicted by (Dawson et al. 2015). On the other hand, the planet size-metallicity relation inferred for Kepler planets by Schlaufman (2015) is significantly shallower than linear, see Figure 3 Hence, it is clear that the planet-metallicity correlation predicted by In Situ formation models is not observed.

In the Planet Migration scenario, the mass of planets that form in outer disk is also dependent on disk metallicity. However, the subsequent inward migration may shape the observed distribution of exoplanets in a different way. Cossou et al. (2014) find that super-earths consistently form in a set of simulations varying the dust-togas ratio, a proxy of metallicity, by a factor 4 . The total mass of planetary systems show an almost linear dependence on metallicity, and hence does not deviate significantly from the predictions of In Situ formation models. Coleman and Nelson (2016) model the growth and migration of super-earths, and find that planets in low-metallicity disks do not reach the mass required for efficient inward migration, and hence close-in super-earths do not form. Instead small mobile bodies (pebbles) must play an important role in the formation of super-earths, though the predictions of such a model have not been explored in detail.

Low Mass Stars The anti-correlation between the occurrence of planets at short orbital period and the stellar mass poses an urgent problem for planet formation theories: How to explain the elevated planet occurrence rates of low mass stars if 


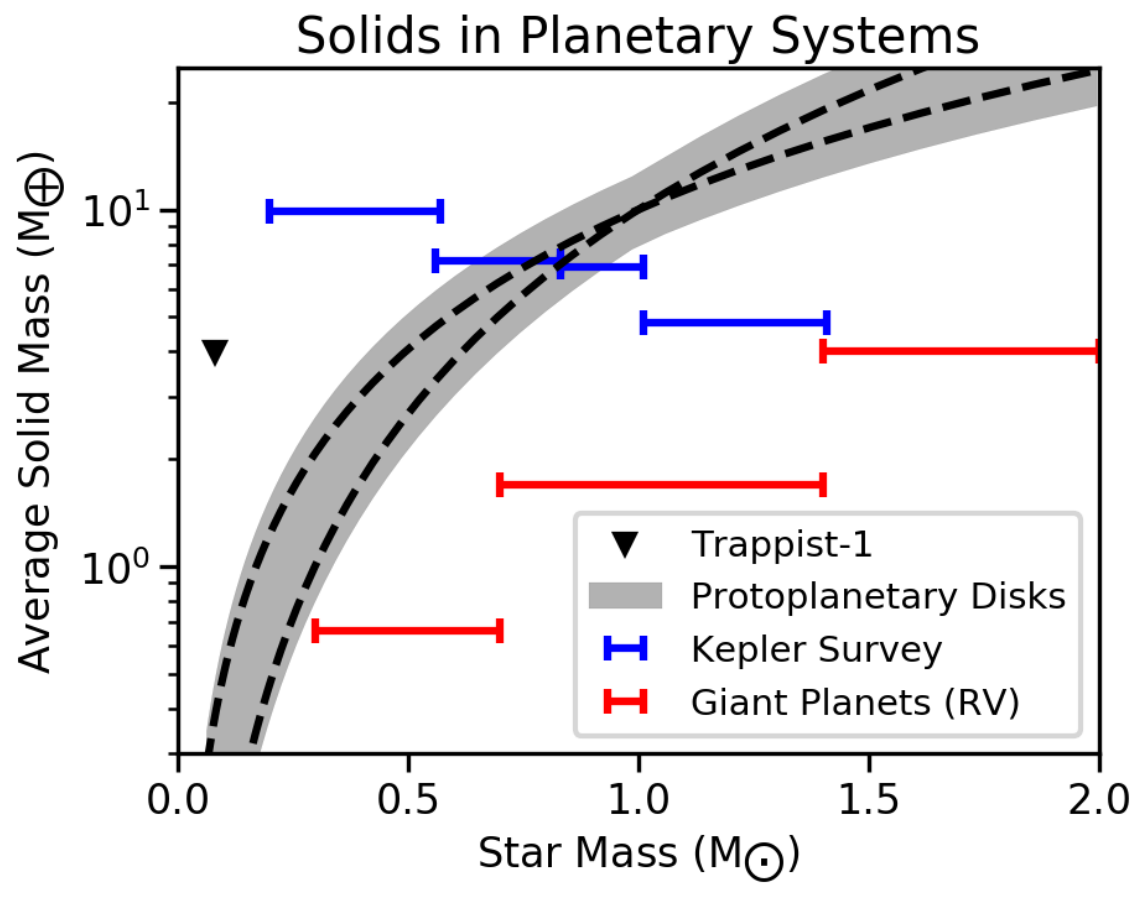

Fig. 7 Average mass in solids around stars of different masses. Blue bars show the average solid mass of planetary systems within an orbital period of 150 days from Mulders et al. (2015c), estimated assuming the mass-radius relation from Wolfgang et al. (2016) and a solid mass of $20 M_{\oplus}$ per giant planet. Red bars show the amount of solids in giant planets out to $\sim 2.5$ au estimated from the occurrence rates in Johnson et al. (2010) and assuming $20 M_{\oplus}$ of solids per planet. The dashed lines shows dust masses of protoplanetary disks in the Chamaeleon I star forming region from Pascucci et al. (2016). The different slopes of the two lines reflect some of the uncertainties in the derived stellar-mass dependence. The black triangle indicates the location of the Trappist-1 planetary system (Gillon et al. 2017) using planet masses from Wang et al. (2017).

less material is available in their disks to form planets? Figure 7 illustrates this issue, showing the estimated amounts of solids around stars of different masses in:

- Protoplanetary disks in the Chamaeleon I star forming region, from Pascucci et al. (2016), probing solids at scales of $\sim 10-100$ au.

- Planets at orbital periods less than 150 days in the Kepler survey, from Mulders et al. (2015c).

- Giant planets out to $2.5 \mathrm{au}$, from Johnson et al. (2010), assuming $20 M_{\oplus}$ of solids per giant planet.

While the solids in giant planets and protoplanetary disks show a positive scaling with stellar mass, this relation breaks down for sub-Neptunes at short orbital periods. The estimated amount of solids in M dwarf planetary systems is higher than that in 


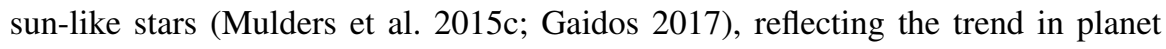
occurrence.

Many studies have assumed a linear dependence of disk solids on stellar mass based on simple scaling arguments, pre-dating a robust determination of such a linear relation (Williams and Cieza 2011; Mohanty et al. 2013, Andrews et al. 2013, e.g.). Recent surveys of star forming regions with ALMA show that the disk massstellar mass relation is in fact even steeper than linear (e.g. Barenfeld et al. 2016, Ansdell et al. 2016, Pascucci et al. 2016) with a power-law index between 1.1-1.9 depending on assumptions of how the disk temperature scales with stellar mass. While millimeter observations probe the solid inventory at scales of $\sim 10-100$ au that are significantly larger than the radial velocity and transit planets populations discussed here, they do confirm the expectation that disk properties scale with stellar mass.

A strict In Situ formation model for sub-Neptunes, where planet mass is directly related to mass available in disk (e.g. Raymond et al. 2007; Ciesla et al. 2015), is not favored by stellar-mass dependencies as evident from Figure 7. Radial redistribution of material likely plays a role, as illustrated in Figure 8 There is some evidence in the exoplanet population that the solid distribution in M dwarf disk may have been more centrally peaked than for sun-like stars (Gaidos 2017), perhaps reflecting more efficient radial drift of dust around low-mass stars (Pinilla et al.2013). Besides disk mass, the lower luminosity of less massive stars (both on the main sequence and pre-main-sequence) means that disk are cooler than their counterparts around higher mass stars. Structural differences in the density and temperature of disks around lower-mass stars move the location of characteristic radii that may play a role in the formation of planets, such as the location of an inner cavity (Lee and Chiang 2017), sublimation fronts of silicates (Boley et al. 2014) and ices ("snow line") (Mulders et al. 2015a), regions of outward planet migration, and dead zone boundaries (Hasegawa and Pudritz 2010). Because these radii do not directly impact the surface density of solids but may affect planet trapping and migration, it is tempting to point to planet migration as origin of the observed trends. However, as noted before, it is not clear how an enhancement of the inner disk density by drift of solids that precedes the giant impact phase would impact the planet population.

Stellar mass dependencies in the planet formation and migration process have been investigated by a number of studies. Planet population synthesis models with a reduced type-I migration rate produce more small planets around $\mathrm{M}$ dwarfs, because Neptune-mass planets can migrate in through type-II migration in low-mass stars (Alibert et al. 2011; Ida and Lin 2005). However, because of the reduced type-I migration rate, the predicted planet populations are not consistent with the observed occurrence of sub-Neptunes (e.g. Howard et al. 2012). Recent models include more realistic descriptions of type-I migration (Alibert et al. 2013; Dittkrist et al.|2014), but it is not yet clear how they affect the distribution of planets around lower mass stars.

Hasegawa and Pudritz (2013) model planet formation and migration around stars ranging in mass from $0.5 M_{\odot}$ to $1.5 M_{\odot}$, investigating the effect of stellar-mass dependent disk structure on type-I migration. They find that the population of close-in 


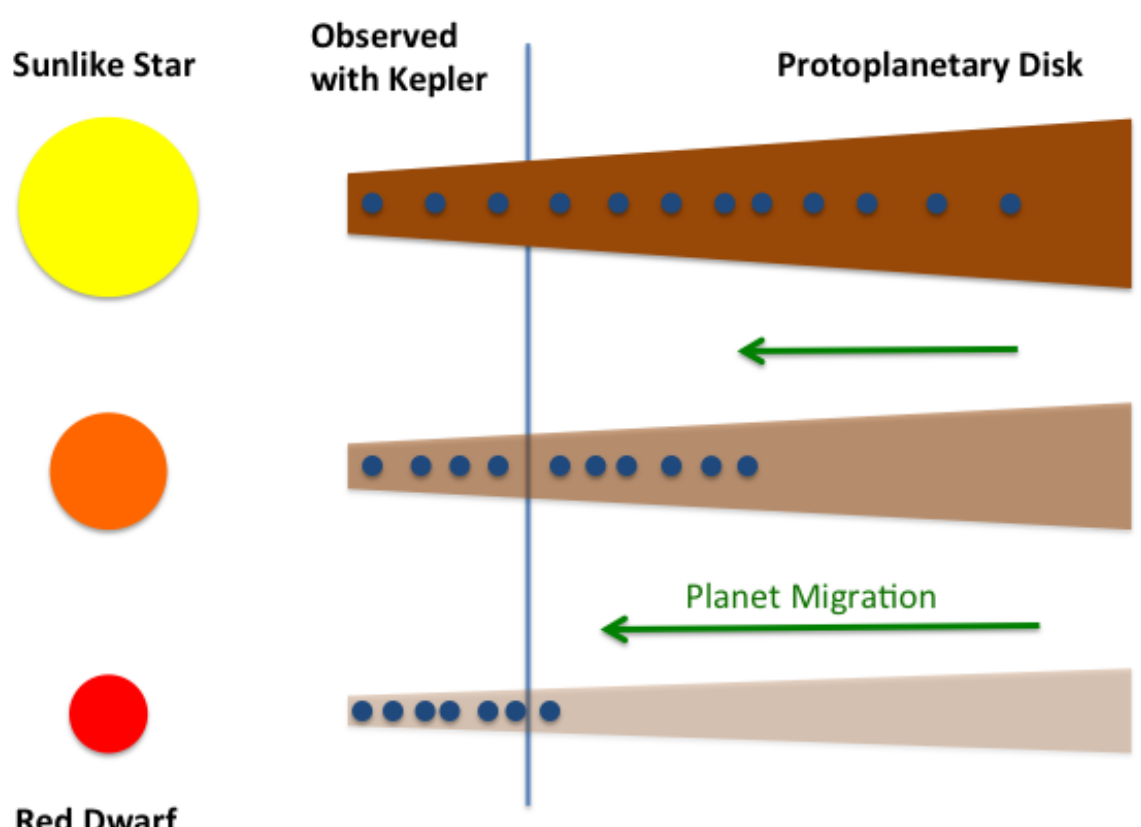

\section{Red Dwarf}

Fig. 8 Illustration of stellar-mass dependencies in the exoplanet and protoplanetary disk population. This visualization highlights the role that radial redistribution of solids through inward planet migration or radial drift may play in shaping the observed distribution of planets at short orbital periods, in particular the elevated occurrence of planets around $\mathrm{M}$ dwarfs.

small planets $\left(<0.7 \mathrm{au},<30 M_{\oplus}\right)$ is less sensitive to the stellar mass than the population of giant planets, and even shows an occurrence rate of $\mathrm{M}$ stars that is higher than for solar-mass stars. While this trend is encouraging, the total mass of planetary systems formed is still a strong function of disk mass, with the majority of mass in giant planets at larger orbital periods. It is not clear if these results hold up in simulations that predominantly form sub-Neptunes, as the occurrence rate of giant planets may be too low to explain the elevated occurrence rates around low-mass stars (Mulders et al. $2015 \mathrm{~b}(\mathrm{c})$. Nonetheless, an anti-correlation between close-in planets and long-period giants has been suggested based for different planet formation scenarios (e.g. Izidoro et al. 2015, Batygin and Laughlin 2015)

\section{Future Directions}

The current census of exoplanets is incomplete for very low mass stars, and it is not clear whether the anti-correlation between stellar mass and planet occurrence rate extends to the lowest mass stars. The lowest mass $\mathrm{M}$ dwarfs have been targeted by ground-based exoplanet surveys such as MEarth (Berta et al.|2012) and TRAPPIST 
(Jehin et al.2011). These surveys have yielded spectacular discoveries for exoplanet atmospheric characterization (e.g. Charbonneau et al. 2009; Gillon et al. 2017), but their sample sizes are typically two orders of magnitude smaller than more sensitive space-based surveys. Nonetheless, placing these results in the context of trends identified with Kepler (see Figure 7p will provide new insight into planet formation around low-mass stars.

$\mathrm{K} 2$, the re-purposed Kepler mission, is surveying different regions along the galactic plane, drastically increasing the number of stars surveyed for transiting planets. A large fraction of its target stars are low-mass K and $\mathrm{M}$ dwarfs $(\sim 41 \%$ of all stars in the first two years, Huber et al. 2016). With more than 100 exoplanets discovered and a projected planet yield up to $\sim 1000$ planets (Crossfield et al. 2016), the $\mathrm{K} 2$ mission has the prospect to significantly advance our understanding of the exoplanet population around low-mass stars. Putting these results into context of the exoplanet population studies performed with Kepler will require an estimate of the survey detection efficiency of K2 (e.g. Foreman-Mackey et al. 2015) as well as a reliable estimate of stellar parameters (e.g. Dressing et al. 2017).

TESS, the Transiting Exoplanet Survey Sattelite launched in april 2018, will perform an all-sky survey of transiting planets around bright stars, including $\mathrm{M}$ dwarfs (Ricker et al. 2014). The area of the sky surveyed will be $\sim 400$ larger than the initial Kepler field, though the majority of stars will be surveyed for less than 27 days. Habitable zone transiting planets are only accessible around M stars, which constitute a large fraction of the $\sim 200,000$ TESS target stars (Sullivan et al.|2015). The majority of planets smaller than $2 R_{\oplus}$ are expected to be discovered around $\mathrm{M}$ dwarfs. Combined with estimates of the survey detection efficiency (e.g. Wang et al.2016), TESS will constrain the population of (short-period) exoplanets around M dwarfs.

\section{Conclusions}

Giant planets occur more frequent around more massive and more metal-rich stars. These trends support the core-accretion scenario for giant planet formation in which accretion of a gaseous envelope starts after a sufficiently rapid assembly of a massive rocky core. The threshold for reaching the critical core mass is reached more easily in protoplanetary disks with a larger amount of condensible solids around metal-rich stars and in more massive disks around more massive stars.

These results stand in contrast to the population of exoplanets smaller than Neptune, those that are found in abundance with Kepler and represent the bulk of the exoplanet population. These sub-Neptunes are found around stars with a wide range of metallicities, indicating that planet formation is a robust process that occurs efficiently in a variety of environments. Curiously, sub-Neptunes occur much more frequently around low-mass $\mathrm{M}$ dwarfs than around solar-mass stars, at least at separations much less than an au. Why lower-mass disks around lower mass stars pro- 
duce more planets remains unclear, but may be connected to the inward migration processes in protoplanetary disks that take place during planet formation.

Future and ongoing surveys of transiting exoplanets around lower mass stars, combined with characterization of stars in these surveys, may shed new light on the formation of planets in a wide range of stellar environments.

\section{References}

Adibekyan V, Figueira P Santos NC (2016) Which Type of Planets do We Expect to Observe in the Habitable Zone? Origins of Life and Evolution of Biospheres 46(4):351-359

Adibekyan VZ, Figueira P, Santos NC et al. (2013) Orbital and physical properties of planets and their hosts: new insights on planet formation and evolution. Astronomy \& Astrophysics 560:A51

Alibert Y, Mordasini C Benz W (2011) Extrasolar planet population synthesis. III. Formation of planets around stars of different masses. Astronomy \& Astrophysics 526:A63

Alibert Y, Carron F, Fortier A et al. (2013) Theoretical models of planetary system formation: mass vs. semi-major axis. Astronomy \& Astrophysics 558:A109

Andrews S, Rosenfeld KA, Kraus AL Wilner DJ (2013) The Mass Dependence between Protoplanetary Disks and their Stellar Hosts. The Astrophysical Journal 771(2):129

Ansdell M, Williams JP, van der Marel N et al. (2016) ALMA Survey of Lupus Protoplanetary Disks. I. Dust and Gas Masses. The Astrophysical Journal 828(1):46

Barenfeld SA, Carpenter JM, Ricci L Isella A (2016) ALMA Observations of Circumstellar Disks in the Upper Scorpius OB Association. The Astrophysical Journal 827(2):142

Batygin K Laughlin G (2015) Jupiter's decisive role in the inner Solar System's early evolution. In: Proceedings of the National Academy of Sciences, pp 4214-4217

Bayliss D, Gillen E, Eigmuller P et al. (2017) NGTS-1b: A hot Jupiter transiting an M-dwarf. arXivorg p arXiv: 1710.11099

Beaugé C Nesvorný D (2013) Emerging Trends in a Period-Radius Distribution of Close-in Planets. The Astrophysical Journal 763(1):12

Berta ZK, Irwin J, Charbonneau D, Burke CJ Falco EE (2012) Transit Detection in the MEarth Survey of Nearby M Dwarfs: Bridging the Clean-first, Search-later Divide. The Astronomical Journal 144(5): 145

Boley AC, Morris MA Ford EB (2014) Overcoming the Meter Barrier and the Formation of Systems with Tightly Packed Inner Planets (STIPs). The Astrophysical Journal Letters 792(2):L27

Bonfils X, Mayor M, Delfosse X et al. (2007) The HARPS search for southern extra-solar planets. X. A m sin $i=11$ Mearth planet around the nearby spotted M dwarf GJ 674. Astronomy \& Astrophysics 474(1):293-299

Boss AP (1997) Giant planet formation by gravitational instability. Science 276(5320):1836-1839

Boss AP (2006) Rapid Formation of Gas Giant Planets around M Dwarf Stars. The Astrophysical Journal 643(1):501-508

Brown TM, Latham DW, Everett ME Esquerdo GA (2011) Kepler Input Catalog: Photometric Calibration and Stellar Classification. The Astronomical Journal 142(4):112

Buchhave LA Latham DW (2015) The Metallicities of Stars with and without Transiting Planets. The Astrophysical Journal 808(2):187

Buchhave LA, Batalha N, Latham DW et al. (2012) An abundance of small exoplanets around stars with a wide range of metallicities. Nature 486(7):375-377

Buchhave LA, Bizzarro M, Latham DW et al. (2014) Three regimes of extrasolar planet radius inferred from host star metallicities. Nature 509(7):593-595

Burke CJ, Christiansen JL, Mullally F et al. (2015) Terrestrial Planet Occurrence Rates for the Kepler GK Dwarf Sample. The Astrophysical Journal 809(1):8 
Butler RP, Johnson JA, Marcy GW et al. (2006) A Long-Period Jupiter-Mass Planet Orbiting the Nearby M Dwarf GJ 849. The Publications of the Astronomical Society of the Pacific 118(8):1685-1689

Chambers JE Wetherill GW (1998) Making the Terrestrial Planets: N-Body Integrations of Planetary Embryos in Three Dimensions. Icarus 136(2):304-327

Charbonneau D, Berta ZK, Irwin J et al. (2009) A super-Earth transiting a nearby low-mass star. Nature 462(7):891-894

Chiang E Laughlin G (2013) The minimum-mass extrasolar nebula: in situ formation of close-in super-Earths. Monthly Notices of the Royal Astronomical Society 431(4):3444-3455

Christiansen JL, Clarke BD, Burke CJ et al. (2015) Measuring Transit Signal Recovery in the Kepler Pipeline II: Detection Efficiency as Calculated in One Year of Data. The Astrophysical Journal 810(2):95

Ciesla FJ, Mulders GD, Pascucci I Apai D (2015) Volatile Delivery to Planets from Water-rich Planetesimals around Low Mass Stars. The Astrophysical Journal 804(1):9

Clanton C Gaudi BS (2014) Synthesizing Exoplanet Demographics from Radial Velocity and Microlensing Surveys. II. The Frequency of Planets Orbiting M Dwarfs. The Astrophysical Journal 791(2):91

Coleman GAL Nelson RP (2016) On the formation of compact planetary systems via concurrent core accretion and migration. Monthly Notices of the Royal Astronomical Society 457(3):2480-2500

Cossou C, Raymond SN, Hersant F Pierens A (2014) Hot super-Earths and giant planet cores from different migration histories. Astronomy \& Astrophysics 569:A56

Courcol B, Bouchy F Deleuil M (2016) An upper boundary in the mass-metallicity plane of exoNeptunes. Monthly Notices of the Royal Astronomical Society 461(2):1841-1849

Crossfield IJM, Ciardi DR, Petigura EA et al. (2016) 197 Candidates and 104 Validated Planets in K2's First Five Fields. The Astrophysical Journal Supplement Series 226(1):7

Cumming A, Butler RP, Marcy GW et al. (2008) The Keck Planet Search: Detectability and the Minimum Mass and Orbital Period Distribution of Extrasolar Planets. Publications of the Astronomical Society of Pacific 120(8):531-

Dawson RI, Chiang E Lee EJ (2015) A metallicity recipe for rocky planets. Monthly Notices of the Royal Astronomical Society 453(2):1471-1483

Dittkrist KM, Mordasini C, Klahr H, Alibert Y Henning T (2014) Impacts of planet migration models on planetary populations. Effects of saturation, cooling and stellar irradiation. Astronomy \& Astrophysics 567:A121

Dong S Zhu Z (2013) Fast Rise of "Neptune-size" Planets (4-8 R $Ł$ ) from P 10 to 250 Days-Statistics of Kepler Planet Candidates up to 0.75 AU. The Astrophysical Journal 778(1):53

Dong S, Zheng Z, Zhu Z et al. (2014) On the Metallicities of Kepler Stars. The Astrophysical Journal Letters 789(1):L3

Dong S, Xie JW, Zhou JL, Zheng Z Luo A (2017) LAMOST Reveals Neptune-size Cousins of hot Jupiters, preferentially in (metal-)rich and one-child Kepler families. arXivorg $p$ arXiv: 1706.07807

Dressing C Charbonneau D (2013) The Occurrence Rate of Small Planets around Small Stars. The Astrophysical Journal 767(1):95

Dressing C Charbonneau D (2015) The Occurrence of Potentially Habitable Planets Orbiting M Dwarfs Estimated from the Full Kepler Dataset and an Empirical Measurement of the Detection Sensitivity. The Astrophysical Journal 807(1):45

Dressing C, Newton ER, Schlieder JE et al. (2017) Characterizing K2 Candidate Planetary Systems Orbiting Low-mass Stars. I. Classifying Low-mass Host Stars Observed during Campaigns 1-7. The Astrophysical Journal 836(2):167

Endl M, Cochran WD, Tull RG MacQueen PJ (2003) A Dedicated M Dwarf Planet Search Using The Hobby-Eberly Telescope. The Astronomical Journal 126(6):3099-3107

Endl M, Cochran WD, Kürster M et al. (2006) Exploring the Frequency of Close-in Jovian Planets around M Dwarfs. The Astrophysical Journal 649(1):436-443 
Everett ME, Howell SB, Silva DR Szkody P (2013) Spectroscopy of Faint Kepler Mission Exoplanet Candidate Host Stars. The Astrophysical Journal 771(2):107

Fabrycky DC, Lissauer JJ, Ragozzine D et al. (2014) Architecture of Kepler's Multi-transiting Systems. II. New Investigations with Twice as Many Candidates. The Astrophysical Journal 790(2): 146

Fischer DA Valenti J (2005) The Planet-Metallicity Correlation. The Astrophysical Journal 622(2):1102-1117

Foreman-Mackey D, Montet BT, Hogg DW et al. (2015) A Systematic Search for Transiting Planets in the K2 Data. The Astrophysical Journal 806(2):215

Frasca A, Molenda-Zakowicz J, De Cat P et al. (2016) Activity indicators and stellar parameters of the Kepler targets. An application of the ROTFIT pipeline to LAMOST-Kepler stellar spectra. Astronomy \& Astrophysics 594:A39

Fressin F, Torres G, Charbonneau D et al. (2013) The False Positive Rate of Kepler and the Occurrence of Planets. The Astrophysical Journal 766(2):81

Fuhrmann K, Pfeiffer MJ Bernkopf J (1998) F- and G-type stars with planetary companions: upsilon Andromedae, rho (1) Cancri, tau Bootis, 16 Cygni and rho Coronae Borealis. Astronomy \& Astrophysics 336:942-952

Gaidos E (2017) A Minimum Mass Nebula for M Dwarfs. arXivorg p arXiv:1704.03265

Gaidos E Mann AW (2014) M Dwarf Metallicities and Giant Planet Occurrence: Ironing Out Uncertainties and Systematics. The Astrophysical Journal 791(1):54

Gaidos E, Fischer DA, Mann AW Howard AW (2013) An Understanding of the Shoulder of Giants: Jovian Planets around Late K Dwarf Stars and the Trend with Stellar Mass. The Astrophysical Journal 771(1):18

Gaidos E, Mann AW, Kraus AL Ireland M (2016) They are small worlds after all: revised properties of Kepler M dwarf stars and their planets. Monthly Notices of the Royal Astronomical Society 457(3):2877-2899

Ghezzi L, Cunha K, Smith VV et al. (2010) Stellar Parameters and Metallicities of Stars Hosting Jovian and Neptunian Mass Planets: A Possible Dependence of Planetary Mass on Metallicity. The Astrophysical Journal 720(2):1290-1302

Gilliland RL, Brown TM, Guhathakurta P et al. (2000) A Lack of Planets in 47 Tucanae from a Hubble Space Telescope Search. The Astrophysical Journal 545(1):L47-L51

Gillon M, Triaud AHMJ, Demory BO et al. (2017) Seven temperate terrestrial planets around the nearby ultracool dwarf star TRAPPIST-1. Nature 542(7):456-460

Goldreich P Schlichting HE (2014) Overstable Librations can Account for the Paucity of Mean Motion Resonances among Exoplanet Pairs. The Astronomical Journal 147(2):32

Gonzalez G (1997) The stellar metallicity-giant planet connection. Monthly Notices of the Royal Astronomical Society 285(2):403-412

Gonzalez G (1998) Spectroscopic analyses of the parent stars of extrasolar planetary system candidates. Astronomy \& Astrophysics 334:221-238

Gonzalez G, Laws C, Tyagi S Reddy BE (2001) Parent Stars of Extrasolar Planets. VI. Abundance Analyses of 20 New Systems. The Astronomical Journal 121(1):432-452

Guo X, Johnson JA, Mann AW et al. (2016) The metallicity distribution and hot Jupiter rate of the Kepler field: Hectochelle High-resolution spectroscopy for 776 Kepler target stars. eprint arXiv: 161201616

Hansen BMS Murray N (2012) Migration Then Assembly: Formation of Neptune-mass Planets inside 1 AU. The Astrophysical Journal 751(2):158

Hansen BMS Murray N (2013) Testing in Situ Assembly with the Kepler Planet Candidate Sample. The Astrophysical Journal 775(1):53

Hasegawa Y Pudritz RE (2010) Radiatively heated, protoplanetary discs with dead zones - I. Dust settling and thermal structure of discs around M stars. Monthly Notices of the Royal Astronomical Society 401(1):143-159

Hasegawa Y Pudritz RE (2013) Planetary Populations in the Mass-Period Diagram: A Statistical Treatment of Exoplanet Formation and the Role of Planet Traps. The Astrophysical Journal 778(1):78 
Hekker S Melendez J (2007) Precise radial velocities of giant stars. III. Spectroscopic stellar parameters. Astronomy \& Astrophysics 475(3):1003-1009

Howard AW, Marcy GW, Johnson JA et al. (2010) The Occurrence and Mass Distribution of Closein Super-Earths, Neptunes, and Jupiters. Science 330(6):653-655

Howard AW, Marcy GW, Bryson ST et al. (2012) Planet Occurrence within 0.25 AU of Solar-type Stars from Kepler. The Astrophysical Journal Supplement 201(2):15

Huber D, Silva Aguirre V, Matthews JM et al. (2014) Revised Stellar Properties of Kepler Targets for the Quarter 1-16 Transit Detection Run. The Astrophysical Journal Supplement 211(1):2

Huber D, Bryson ST, Haas MR et al. (2016) The K2 Ecliptic Plane Input Catalog (EPIC) and Stellar Classifications of 138,600 Targets in Campaigns 1-8. The Astrophysical Journal Supplement Series 224(1):2

Ida S Lin DNC (2004) Toward a Deterministic Model of Planetary Formation. II. The Formation and Retention of Gas Giant Planets around Stars with a Range of Metallicities. The Astrophysical Journal 616(1):567-572

Ida S Lin DNC (2005) Toward a Deterministic Model of Planetary Formation. III. Mass Distribution of Short-Period Planets around Stars of Various Masses. The Astrophysical Journal 626(2):1045-1060

Ida S Lin DNC (2008) Toward a Deterministic Model of Planetary Formation. IV. Effects of Type I Migration. The Astrophysical Journal 673(1):487-501

Ikoma M, Nakazawa K Emori H (2000) Formation of Giant Planets: Dependences on Core Accretion Rate and Grain Opacity. The Astrophysical Journal 537(2):1013-1025

Izidoro A, Raymond SN, Morbidelli A, Hersant F Pierens A (2015) Gas Giant Planets as Dynamical Barriers to Inward-Migrating Super-Earths. The Astrophysical Journal Letters 800(2):L22

Izidoro A, Ogihara M, Raymond SN et al. (2017) Breaking the Chains: Hot Super-Earth systems from migration and disruption of compact resonant chains. arXivorg p arXiv:1703.03634

Jehin E, Gillon M, Queloz D et al. (2011) TRAPPIST: TRAnsiting Planets and PlanetesImals Small Telescope. The Messenger 145:2-6

Jofré E, Petrucci R, Saffe C et al. (2015) Stellar parameters and chemical abundances of 223 evolved stars with and without planets. Astronomy \& Astrophysics 574:A50

Johnson JA Apps K (2009) On the Metal Richness of M Dwarfs with Planets. The Astrophysical Journal 699(2):933-937

Johnson JA, Butler RP, Marcy GW et al. (2007) A New Planet around an M Dwarf: Revealing a Correlation between Exoplanets and Stellar Mass. The Astrophysical Journal 670(1):833-840

Johnson JA, Aller KM, Howard AW Crepp JR (2010) Giant Planet Occurrence in the Stellar MassMetallicity Plane. Publications of the Astronomical Society of Pacific 122(8):905-915

Johnson JA, Petigura EA, Fulton BJ et al. (2017) The California-Kepler Survey. II. Precise Physical Properties of 2025 Kepler Planets and Their Host Stars. arXivorg p arXiv: 1703.10402

Jones MI, Jenkins JS, Brahm R et al. (2016) Four new planets around giant stars and the massmetallicity correlation of planet-hosting stars. Astronomy \& Astrophysics 590:A38

Kennedy G Kenyon SJ (2008) Planet Formation around Stars of Various Masses: The Snow Line and the Frequency of Giant Planets. The Astrophysical Journal 673(1):502-512

Kokubo E Ida S (1998) Oligarchic Growth of Protoplanets. Icarus 131(1):171-178

Kokubo E Ida S (2000) Formation of Protoplanets from Planetesimals in the Solar Nebula. Icarus 143(1):15-27

Kokubo E Ida S (2002) Formation of Protoplanet Systems and Diversity of Planetary Systems. The Astrophysical Journal 581(1):666-680

Kornet K, Bodenheimer P, Rozyczka M Stepinski TF (2005) Formation of giant planets in disks with different metallicities. Astronomy \& Astrophysics 430(3):1133-1138

Laughlin G Adams FC (1997) Possible Stellar Metallicity Enhancements from the Accretion of Planets. The Astrophysical Journal 491(1):L51-L54

Laughlin G, Bodenheimer P Adams FC (2004) The Core Accretion Model Predicts Few JovianMass Planets Orbiting Red Dwarfs. The Astrophysical Journal 612(1):L73-L76

Laws C, Gonzalez G, Walker KM et al. (2003) Parent Stars of Extrasolar Planets. VII. New Abundance Analyses of 30 Systems. The Astronomical Journal 125(5):2664-2677 
Lee EJ Chiang E (2017) Magnetospheric Truncation, Tidal Inspiral, and the Creation of Shortperiod and Ultra-short-period Planets. The Astrophysical Journal 842(1):40

Lin DNC (2008) Toward a Deterministic Model of Planetary Formation. V. Accumulation Near the Ice Line and Super-Earths. The Astrophysical Journal 685(1):584-595

Lissauer JJ (1987) Timescales for planetary accretion and the structure of the protoplanetary disk. Icarus (ISSN 0019-1035) 69:249-265

Maldonado J, Villaver E Eiroa C (2013) The metallicity signature of evolved stars with planets. Astronomy \& Astrophysics 554:A84

Mamajek EE (2009) Initial Conditions of Planet Formation: Lifetimes of Primordial Disks. In: EXOPLANETS AND DISKS: THEIR FORMATION AND DIVERSITY: Proceedings of the International Conference. AIP Conference Proceedings, Department of Physics and Astronomy, University of Rochester, Rochester, NY 14627-0171, AIP, pp 3-10

Mann AW, Gaidos E, Kraus A Hilton EJ (2013) Testing the Metal of Late-type Kepler Planet Hosts with Iron-clad Methods. The Astrophysical Journal 770(1):43

Marcy GW, Butler RP, Williams E et al. (1997) The Planet around 51 Pegasi. The Astrophysical Journal 481(2):926-935

Mathur S, Huber D, Batalha N et al. (2017) Revised Stellar Properties of Kepler Targets for the Q1-17 (DR25) Transit Detection Run. The Astrophysical Journal Supplement Series 229(2):30

Mayor M Queloz D (1995) A Jupiter-mass companion to a solar-type star. Nature 378(6):355-359

Mayor M, Bonfils X, Forveille T et al. (2009) The HARPS search for southern extra-solar planets. XVIII. An Earth-mass planet in the GJ 581 planetary system. Astronomy \& Astrophysics 507(1):487-494

Mayor M, Marmier M, Lovis C et al. (2011) The HARPS search for southern extra-solar planets XXXIV. Occurrence, mass distribution and orbital properties of super-Earths and Neptunemass planets. arXivorg $\mathrm{p}$ arXiv: 1109.2497

Meru F Bate MR (2010) Exploring the conditions required to form giant planets via gravitational instability in massive protoplanetary discs. Monthly Notices of the Royal Astronomical Society 406(4):2279-2288

Mohanty S, Greaves J, Mortlock D et al. (2013) Protoplanetary Disk Masses from Stars to Brown Dwarfs. The Astrophysical Journal 773(2):168

Montet BT, Crepp JR, Johnson JA, Howard AW Marcy GW (2014) The TRENDS High-contrast Imaging Survey. IV. The Occurrence Rate of Giant Planets around M Dwarfs. The Astrophysical Journal 781(1):28

Mordasini C, Alibert Y Benz W (2009a) Extrasolar planet population synthesis. I. Method, formation tracks, and mass-distance distribution. Astronomy \& Astrophysics 501(3):1139-1160

Mordasini C, Alibert Y, Benz W Naef D (2009b) Extrasolar planet population synthesis. II. Statistical comparison with observations. Astronomy \& Astrophysics 501(3):1161-1184

Mortier A, Santos NC, Sozzetti A et al. (2012) The frequency of giant planets around metal-poor stars. Astronomy \& Astrophysics 543:A45

Mortier A, Santos NC, Sousa S et al. (2013a) On the functional form of the metallicity-giant planet correlation. Astronomy \& Astrophysics 551:A112

Mortier A, Santos NC, Sousa SG et al. (2013b) New and updated stellar parameters for 71 evolved planet hosts. On the metallicity-giant planet connection. Astronomy \& Astrophysics 557:A70

Morton TD Swift J (2014) The Radius Distribution of Planets around Cool Stars. The Astrophysical Journal 791(1):10

Mulders GD, Ciesla FJ, Min M Pascucci I (2015a) The Snow Line in Viscous Disks around Lowmass Stars: Implications for Water Delivery to Terrestrial Planets in the Habitable Zone. The Astrophysical Journal 807(1):9

Mulders GD, Pascucci I Apai D (2015b) A Stellar-mass-dependent Drop in Planet Occurrence Rates. The Astrophysical Journal 798(2):112

Mulders GD, Pascucci I Apai D (2015c) An Increase in the Mass of Planetary Systems around Lower-mass Stars. The Astrophysical Journal 814(2):130

Mulders GD, Pascucci I, Apai D, Frasca A Molenda-Zakowicz J (2016) A Super-solar Metallicity for Stars with Hot Rocky Exoplanets. The Astronomical Journal 152(6):187 
Neves V, Bonfils X, Santos NC et al. (2013) Metallicity of M dwarfs. III. Planet-metallicity and planet-stellar mass correlations of the HARPS GTO M dwarf sample. Astronomy \& Astrophysics 551:A36

Obermeier C, Koppenhoefer J, Saglia RP et al. (2016) Pan-Planets: Searching for hot Jupiters around cool dwarfs. Astronomy \& Astrophysics 587:A49

Pascucci I, Testi L, Herczeg GJ et al. (2016) A Steeper than Linear Disk Mass-Stellar Mass Scaling Relation. The Astrophysical Journal 831(2):125

Pasquini L, Döllinger MP, Weiß A et al. (2007) Evolved stars suggest an external origin of the enhanced metallicity in planet-hosting stars. Astronomy \& Astrophysics 473(3):979-982

Petigura EA, Marcy GW Howard AW (2013) A Plateau in the Planet Population below Twice the Size of Earth. The Astrophysical Journal 770(1):69

Petigura EA, Marcy GW, Winn JN et al. (2017a) The California-Kepler Survey. IV. Metal-rich Stars Host a Greater Diversity of Planets. arXivorg p arXiv: 1712.04042

Petigura EA, Sinukoff E, Lopez ED et al. (2017b) Four Sub-Saturns with Dissimilar Densities: Windows into Planetary Cores and Envelopes. The Astronomical Journal 153(4):142

Pinilla P, Birnstiel T, Benisty M et al. (2013) Explaining millimeter-sized particles in brown dwarf disks. Astronomy \& Astrophysics 554:A95

Pollack JB, Hubickyj O, Bodenheimer P et al. (1996) Formation of the Giant Planets by Concurrent Accretion of Solids and Gas. Icarus 124(1):62-85

Raymond SN, Scalo J Meadows VS (2007) A Decreased Probability of Habitable Planet Formation around Low-Mass Stars. The Astrophysical Journal 669(1):606-614

Raymond SN, Barnes R Mandell AM (2008) Observable consequences of planet formation models in systems with close-in terrestrial planets. Monthly Notices of the Royal Astronomical Society 384(2):663-674

Reffert S, Bergmann C, Quirrenbach A, Trifonov T Künstler A (2015) Precise radial velocities of giant stars. VII. Occurrence rate of giant extrasolar planets as a function of mass and metallicity. Astronomy \& Astrophysics 574:A116

Reid IN (2002) On the Nature of Stars with Planets. The Publications of the Astronomical Society of the Pacific 114(7):306-329

Rein H (2012) Period ratios in multiplanetary systems discovered by Kepler are consistent with planet migration. Monthly Notices of the Royal Astronomical Society: Letters 427(1):L21L24

Ricker GR, Winn JN, Vanderspek R et al. (2014) Transiting Exoplanet Survey Satellite (TESS). In: Oschmann JM, Clampin M, Fazio GG MacEwen HA (eds) Proceedings of the SPIE, Massachusetts Institute of Technology (United States), SPIE, pp 914,320-914,320-15

Rojas-Ayala B, Covey KR, Muirhead PS Lloyd JP (2012) Metallicity and Temperature Indicators in M Dwarf K-band Spectra: Testing New and Updated Calibrations with Observations of 133 Solar Neighborhood M Dwarfs. The Astrophysical Journal 748(2):93

Sadakane K, Ohkubo M, Takeda Y et al. (2002) Abundance Analyses of 12 Parent Stars of Extrasolar Planets Observed with the SUBARU/HDS. Publications of the Astronomical Society of Japan 54(6):911-931

Santos NC, Israelian G Mayor M (2000) Chemical analysis of 8 recently discovered extra-solar planet host stars. Astronomy \& Astrophysics 363:228-238

Santos NC, Israelian G Mayor M (2001) The metal-rich nature of stars with planets. Astronomy \& Astrophysics 373(3):1019-1031

Santos NC, Israelian G, Mayor M, Rebolo R Udry S (2003) Statistical properties of exoplanets. II. Metallicity, orbital parameters, and space velocities. Astronomy \& Astrophysics 398(1):363376

Santos NC, Israelian G Mayor M (2004) Spectroscopic [Fe/H] for 98 extra-solar planet-host stars. Exploring the probability of planet formation. Astronomy \& Astrophysics 415(3):1153-1166

Santos NC, Mayor M, Bonfils X et al. (2011) The HARPS search for southern extrasolar planets. XXV. Results from the metal-poor sample. Astronomy \& Astrophysics 526:A112

Schlaufman KC (2015) A Continuum of Planet Formation between 1 and 4 Earth Radii. The Astrophysical Journal Letters 799(2):L26 
Schlaufman KC Laughlin G (2010) A physically-motivated photometric calibration of M dwarf metallicity. Astronomy \& Astrophysics 519:A105

Schlaufman KC Laughlin G (2011) Kepler Exoplanet Candidate Host Stars Are Preferentially Metal Rich. The Astrophysical Journal 738(2):177

Schlichting HE (2014) Formation of Close in Super-Earths and Mini-Neptunes: Required Disk Masses and their Implications. The Astrophysical Journal Letters 795(1):L15

Silburt A, Gaidos E Wu Y (2015) A Statistical Reconstruction of the Planet Population around Kepler Solar-type Stars. The Astrophysical Journal 799(2):180

Sousa SG, Santos NC, Mayor M et al. (2008) Spectroscopic parameters for 451 stars in the HARPS GTO planet search program. Stellar $[\mathrm{Fe} / \mathrm{H}]$ and the frequency of exo-Neptunes. Astronomy \& Astrophysics 487(1):373-381

Sousa SG, Santos NC, Israelian G, Mayor M Udry S (2011) Spectroscopic stellar parameters for 582 FGK stars in the HARPS volume-limited sample. Revising the metallicity-planet correlation. Astronomy \& Astrophysics 533:A141

Sullivan PW, Winn JN, Berta-Thompson ZK et al. (2015) The Transiting Exoplanet Survey Satellite: Simulations of Planet Detections and Astrophysical False Positives. The Astrophysical Journal 809(1):77

Swift JJ, Johnson JA, Morton TD et al. (2013) Characterizing the Cool KOIs. IV. Kepler-32 as a Prototype for the Formation of Compact Planetary Systems throughout the Galaxy. The Astrophysical Journal 764(1):105

Takeda Y, Sato B Murata D (2008) Stellar Parameters and Elemental Abundances of Late-G Giants. Publications of the Astronomical Society of Japan 60(4):781-802

Terquem C Papaloizou J (2007) Migration and the Formation of Systems of Hot Super-Earths and Neptunes. The Astrophysical Journal 654(2):1110-1120

Terrien RC, Mahadevan S, Bender CF et al. (2012) An H-band Spectroscopic Metallicity Calibration for M Dwarfs. The Astrophysical Journal Letters 747(2):L38

Thommes EW, Matsumura S Rasio FA (2008) Gas Disks to Gas Giants: Simulating the Birth of Planetary Systems. Science 321(5):814-

Udry S Santos NC (2007) Statistical Properties of Exoplanets. Annual Review of Astronomy and Astrophysics 45(1):397-439

Udry S, Mayor M, Benz W et al. (2006) The HARPS search for southern extra-solar planets. V. A 14 Earth-masses planet orbiting HD4308. Astronomy \& Astrophysics 447(1):361-367

Wang D, Hogg DW, Foreman-Mackey D Schölkopf B (2016) A Causal, Data-driven Approach to Modeling the Kepler Data. Publications of the Astronomical Society of Pacific 128(9):094,503-

Wang J Fischer DA (2015) Revealing a Universal Planet-Metallicity Correlation for Planets of Different Sizes Around Solar-type Stars. The Astronomical Journal 149(1):14

Wang S, Wu DH Barclay T (2017) Updated Masses for the TRAPPIST-1 Planets. arXivorg p arXiv: 1704.04290

Ward WR (1997) Protoplanet Migration by Nebula Tides. Icarus 126(2):261-281

Wetherill GW (1985) Occurrence of giant impacts during the growth of the terrestrial planets. Science (ISSN 0036-8075) 228(4701):877-879

Williams JP Cieza LA (2011) Protoplanetary Disks and Their Evolution. Annual Review of Astronomy and Astrophysics 49:67-117

Wilson RF, Teske J, Majewski SR et al. (2017) Elemental Abundances of Kepler Objects of Interest in APOGEE. I. Two Distinct Orbital Period Regimes Inferred from Host Star Iron Abundances. arXivorg p arXiv: 1712.01198

Winn JN, Sanchis-Ojeda R, Rogers L et al. (2017) Absence of a Metallicity Effect for Ultra-shortperiod Planets. The Astronomical Journal 154(2):60

Wittenmyer RA, Jones MI, Zhao J et al. (2017) The Pan-Pacific Planet Search. VI. Giant Planets Orbiting HD 86950 and HD 222076. The Astronomical Journal 153(2):51

Wolfgang A, Rogers LA Ford EB (2016) Probabilistic Mass-Radius Relationship for Sub-NeptuneSized Planets. The Astrophysical Journal 825(1):19

Youdin AN (2011) The Exoplanet Census: A General Method Applied to Kepler. The Astrophysical Journal 742(1):38 
Zhu W, Wang J Huang C (2016) Dependence of Small Planet Frequency on Stellar Metallicity Hidden by Their Prevalence. The Astrophysical Journal 832(2):196 